\title{
Three-color vector nematicon
}

\author{
Urszula A. Laudyn ${ }^{* 1}$, Michał Kwaśny ${ }^{1}$, Mirosław A. Karpierz ${ }^{1}$, \\ and Gaetano Assanto ${ }^{2,3}$ \\ ${ }^{1}$ Faculty of Physics, Warsaw University of Technology, Koszykowa 75, 00-662 Warszawa, \\ ${ }^{2}$ Nonlinear Optics and Opto-Electronics Laboratory, University "Roma Tre", Rome 00146, Italy, \\ ${ }^{3}$ Photonics Lab, Department of Physics, Tampere University of Technology, 33101 Tampere - Finland.
}

Received March 13, 2017; accepted May 4, 2017; published June 30, 2017

\begin{abstract}
Light localization via reorientation in nematic liquid crystals supports multi-component optical spatial solitons, i.e., vector nematicons. By launching three optical beams of different wavelengths and the same input polarization in a bias-free planar cell, we demonstrate a three-color vector nematicon which is self-trapped thanks to its incoherent nature.
\end{abstract}

Optical spatial solitons are known to be stable in nonlocal self-focusing media, such as photorefractive crystals, soft-matter and nematic liquid crystals (NLCs) [1-3]. NLCs are organic liquids with a large degree of angular orientation, so they behave optically as positive uniaxial crystals, with giant reorientational optical nonlinearity, large electro-optic response and high nonlocality. When a light beam with electric field $\mathbf{E}$ propagates in NLC, the induced dipoles tend to undergo the action of a torque

$$
\boldsymbol{\Gamma}=\varepsilon_{0}\left(n_{e}^{2}-n_{o}^{2}\right)(\mathbf{n} \cdot \mathbf{E})(\mathbf{n} \times \mathbf{E}),
$$

with $\varepsilon_{0}$ the dielectric susceptibility of vacuum, $n_{e}$ and $n_{o}$ the refractive indices for electric fields parallel and orthogonal to the optic axis $\mathbf{n}$, respectively. The resulting increase of the orientation angle $\theta$ between the wavevector $\boldsymbol{k}$ and $\mathbf{n}$ is responsible for self-focusing through an increase in the extraordinary-wave refractive index

$$
n_{e}(\theta)=\frac{n_{o} n_{e}}{\sqrt{\left(n_{e}^{2}-n_{o}^{2}\right) \sin ^{2} \theta+n_{e}^{2}}} .
$$

When self-focusing balances out diffraction, a spatial soliton is generated, associated to a graded-index waveguide wider that the forming beam itself [4-5].

Spatial solitons in nematic liquid crystals, often referred to as nematicons [4-6], also exhibit an incoherent nature,

\footnotetext{
*E-mail: ulaudyn@if.pw.edu.pl
}

such that they can be launched by injecting spatially incoherent beams [7-9] or optical wavepackets with various spectral components [10-12].

The generation of vector spatial solitons in birefringent uniaxial materials such as NLC by the collinear injection of input beams at different wavelengths encompasses several problems, despite the incoherent combination of their intensities. In vector solitons, in fact, each component can differ for polarization (extraordinary or ordinary eigenwaves), colour (wavelength) and propagating direction, besides transverse profile, input location and curvature, amplitude. Since optical reorientation is more easily accessed with extraordinary waves, i.e., with an electric field in the plane defined by the wavevector and molecular director (or optic axis), hereby we consider copolarized vector components. Moreover, while vector solitons can also be formed with distinct component profiles [13-17] or counterpropagating excitations [18-19], hereby we focus on co-launching Gaussian input beams with approximately equal linear diffraction lengths (i.e. different input beam waist).[20] Therefore, the components of distinct wavelengths essentially differ for refractive indices and birefringence through chromatic dispersion, as well as for birefringent walkoff and elastic constants which rule the reorientational response [21-22]. While refractive changes and elastic constants determine the size of the nonlinear response through molecular reorientation, [23-24] the wavelength-dependent angular walkoff tends to separate the beams in space $[10,25]$, with each individual Poynting vector pointing in a different direction at angle $\delta$ with respect to the corresponding wavevector in the principal plane $\mathbf{n} \boldsymbol{k}$ of propagation, with

$$
\tan \delta(\theta)=\frac{\left(n_{e}^{2}-n_{o}^{2}\right) \sin \theta \cos \theta}{n_{o}^{2}+\left(n_{e}^{2}-n_{o}^{2}\right) \cos ^{2} \theta} .
$$




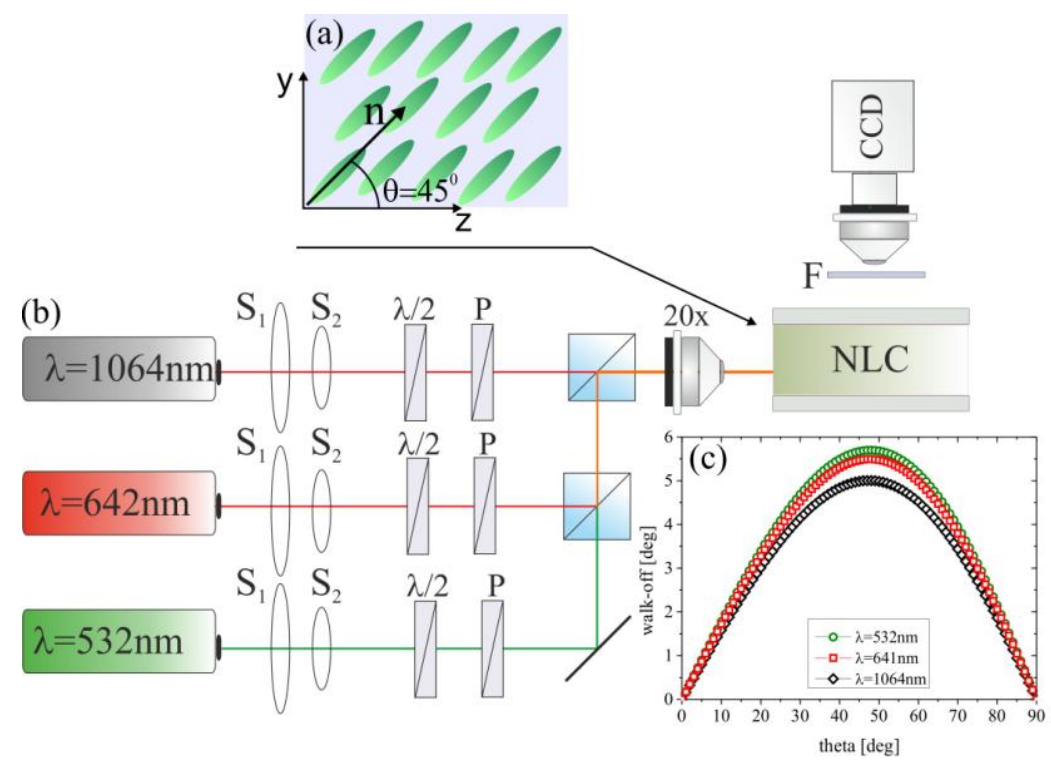

Fig. 1. (a) Planar NLC (bias-free) cell for the study of nematicons. (b) Setup for the generation and observation of three-color nematicons, comprising lenses $S_{1}$ and $S_{2}$ for collimation and waist adjustments, waveplates and polarizers, $20 \times$ microscope objective, beam combiners, dichroic filter and CCD camera. (c) Calculated walk-off angle versus orientation $\theta$ for the three colours.

In the experiments, we employed planar cells comprising two parallel glass slides separated by $50 \mu \mathrm{m}$ and with the interfaces coated with rubbed polyimide layers to anchor the molecular director at $45^{\circ}$ with respect to $z$ in the plane $y z$. The liquid crystals we used was the standard nematic mixture 6CHBT with $n_{o}=1.52$ and $n_{e}=1.68$ at $532 \mathrm{~nm}$; $n_{o}=1.51$ and $n_{e}=1.66$ at $642 \mathrm{~nm}$, and $n_{o}=1.49$ and $n_{e}=1.63$ at $1064 \mathrm{~nm}$, respectively. The cell geometry is sketched in Fig. 1a, the typical setup in Fig. 1b. The calculated walkoff versus orientation angle $\theta$ is graphed in Fig. 1c for three colours. (a)
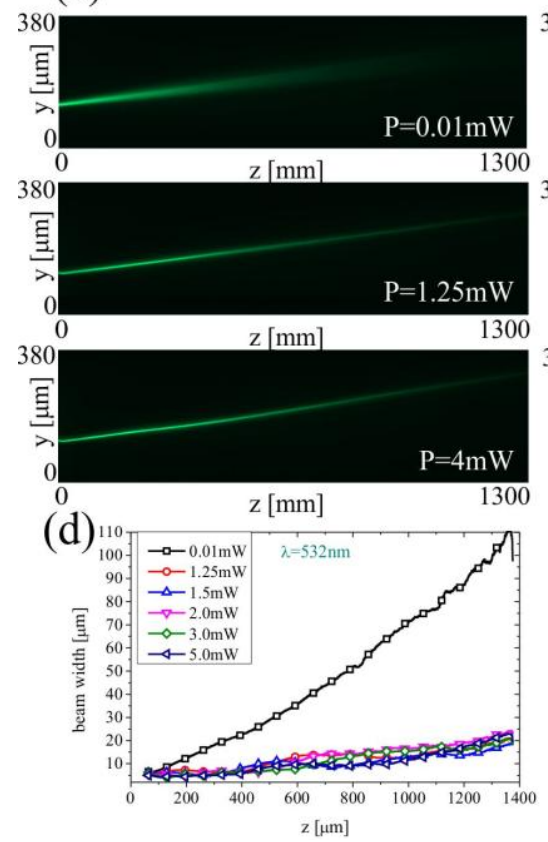

(b)
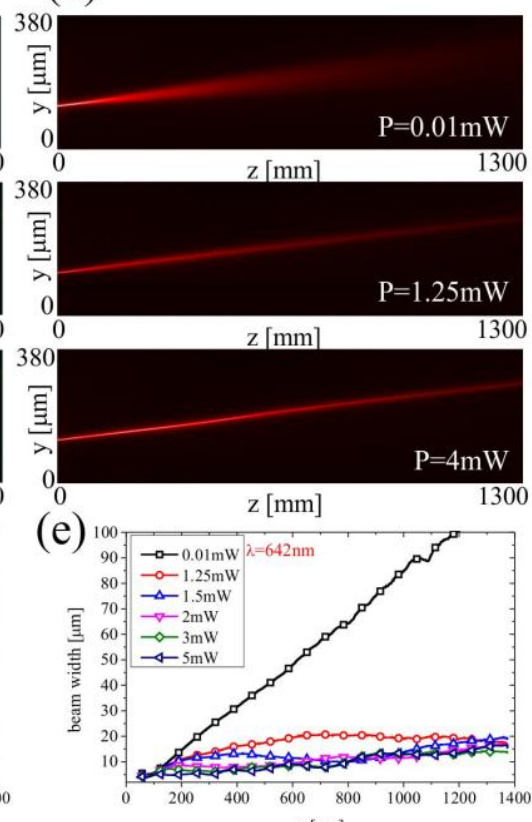

(c)
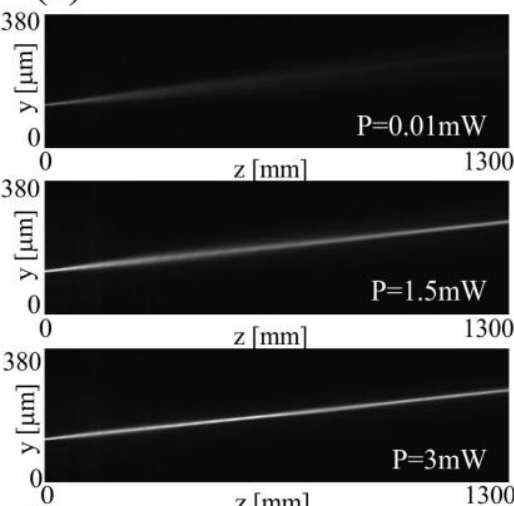

(f)

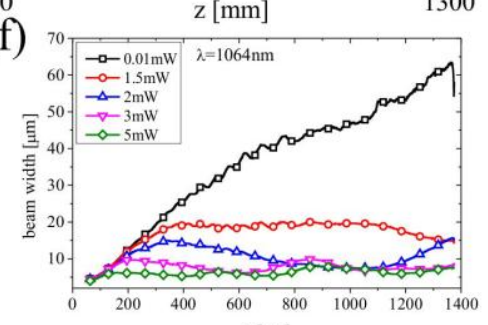

Fig. 2. (a) Extraordinary-wave beam evolution in the observation plane $y z$ at various input powers in the green; (b) same as in (a) for the red; (c) same as in (a) for the near-infrared. (d), (e) and (f) Beam width versus propagation distance $z$ for several input powers, as indicated in the legend. and corresponding to the cases (a), (b) and (c), respectively. 
Nematicons were first generated at each individual wavelength in order to assess the proper launching conditions for each component beam. Figures $2 \mathrm{a}-\mathrm{c}$ show photographs of each beam evolution in the observation plane $y z$ for various input powers. Out-of plane scattering was exploited to image the beam evolution in the plane $y z$ with a CCD camera. The graphs at the bottom, Figs. $2 \mathrm{~d}-\mathrm{f}$ illustrate each beam width versus propagation distance at various input powers as specified in the legend. Width oscillations in the solitary regime are associated to beam breathing in the nonlocal system [26-27].

Figures 3a-c display the three low power component beams in the diffracting regime: clearly, none of them is individually able to self-confine at the given input power. The three copolarized extraordinary-wave beams were launched collinearly by adjusting their input wavevector tilt to make the Poynting vectors overlap. Finally, when launched simultaneously as in Fig. 3(d), their incoherent superposition induced a sufficiently strong potential well to trap all components into a single vector nematicon, with no diffraction over a propagation distance well exceeding $1 \mathrm{~mm}$.

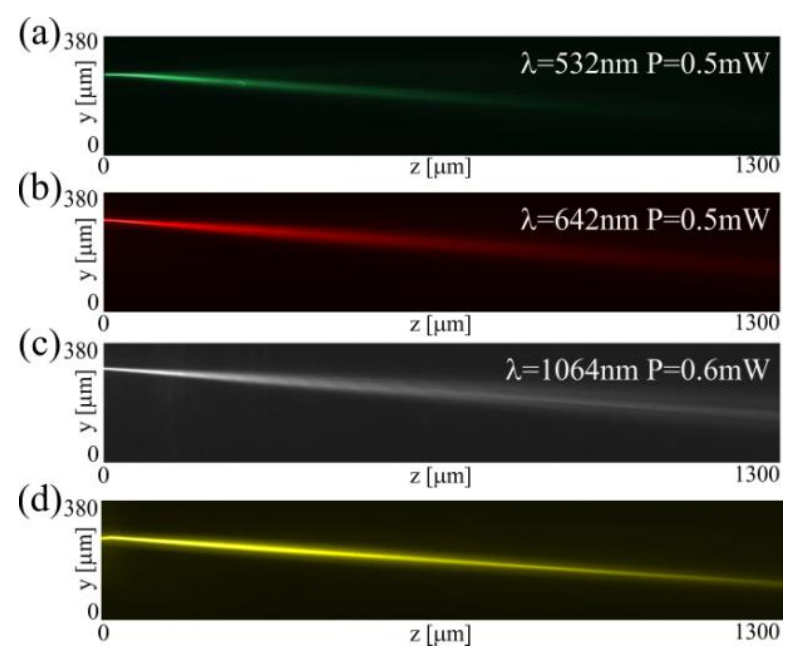

Fig. 3. (a-c) Low power beam diffracting in the sample for a beam at wavelength (a) $532 \mathrm{~nm}$, (b) $642 \mathrm{~nm}$ and (c) $1064 \mathrm{~nm}$, respectively. (d) Three-colour vector nematicon resulting from the simultaneous launch of the components shown above.

In conclusion, we have demonstrated the generation and propagation of incoherent vector nematicons using three colour components, namely red, green and near-infrared beams. The copolarized extraordinary-wave beams, launched collinearly by adjusting their input wavevector tilt to make the Poynting vectors overlap, formed a spatial soliton only when simultaneously present, witnessing the synergistic role of their intensities (powers) through the incoherent nature of the self-induced refractive index potential.
This work was partially supported by the National Centre for Research and Development by the grant agreement LIDER/018/309/L-5/13/NCBR/2014. G.A. gratefully acknowledges support from the Academy of Finland through the Finland Distinguished Professor grant no. 282858 and from the COST Action IC1208 of the European Union.

\section{References}

[1] G.I. Stegeman, M. Segev, Science 286 (5444), 1518 (1999).

[2] W. Królikowski, O. Bang, Phys. Rev. E 63, 016610 (2000).

[3] D. Suter, T. Blasberg, Phys. Rev. A 48, 4583 (1993).

[4] G. Assanto, M. Peccianti, IEEE J. Quantum Electron. 39(1), 13 (2003).

[5] G. Assanto, M. Karpierz, Liq. Cryst. 36(10), 1161 (2009).

[6] M. Peccianti, G. Assanto, Phys. Rep. 516, 147 (2012).

[7] M. Peccianti, G. Assanto, Opt. Lett. 26(22), 1791 (2001).

[8] M. Peccianti, G. Assanto, Phys. Rev. E Rap. Commun. 65, 035603 (2002).

[9] G. Assanto, M. Peccianti, C. Umeton, A. De Luca, I.C. Khoo, Mol. Cryst. Liq. Cryst. 375, 617 (2002).

[10] A. Alberucci, M. Peccianti, G. Assanto, A. Dyadyusha, M. Kaczmarek, Phys. Rev. Lett. 97, 153903 (2006).

[11] G. Assanto, N.F. Smyth, A.L. Worthy, Phys. Rev. A 78(1), 013832 (2008).

[12] G. Assanto, K. Garcia-Reimbert, A.A. Minzoni, N.F. Smyth, A. Worthy, Physica D 240, 1213 (2011).

[13] G. Assanto, A.A. Minzoni, N.F. Smyth, Opt. Lett. 39(3), 509 (2014).

[14] G. Assanto, A.A. Minzoni, N. F. Smyth, Phys. Rev. A 89, 013827 (2014).

[15] G. Assanto, N.F. Smyth, J. Las. Opt. Photon. 1, 105 (2014).

[16] Y.V. Izdebskaya, G. Assanto, W. Krolikowski, Opt. Lett. 40(17), 4182 (2015).

[17] Y.V. Izdebskaya, W. Krolikowski, N.F. Smyth, G. Assanto, J. Opt. 18(5), 054006 (2016).

[18] J.F. Henninot, J. Blach, M. Warenghem, J. Opt. A 9, 20 (2007).

[19] Y.V. Izdebskaya, V.G. Shvedov, A.S. Desyatnikov, W.Z. Krolikowski, M. Belic, G. Assanto, Y.S. Kivshar, Opt. Express 18 (4), 3258 (2010).

[20] N. Karimi, A. Alberucci, M. Virkki, M. Kauranen, G. Assanto, J. Opt. Soc. Am. B 5 (33), 903 (2016).

[21] P.G. de Gennes, J. Prost, The Physics of Liquid Crystals, Oxford Science Publications (Clarendon Press, 2nd edition, 1993).

[22] I.C. Khoo, Liquid Crystals: Physical Properties and Nonlinear Optical Phenomena (Wiley, New York, 1995).

[23] A. Piccardi, M. Trotta, M. Kwasny, A. Alberucci, R. Asquini, M. Karpierz, A. d'Alessandro, G. Assanto, Appl. Phys. B 104(4), 805 (2011).

[24] M. Kwasny, U.A. Laudyn, F.A. Sala, A. Alberucci, M.A. Karpierz, G. Assanto, Phys. Rev. A 86(1), 01382 (2012).

[25] M. Peccianti, A. Fratalocchi, G. Assanto, Opt. Express 12(26), 6524 (2004).

[26] C. Conti, M. Peccianti, G. Assanto, Phys. Rev. Lett. 92(11), 113902 (2004).

[27] A. Alberucci, C.-P. Jisha, G. Assanto, J. Opt. 18, 125501 (2016). 